\title{
5.3 TRANSFER OF ENERGY TO THE CRAB NEBULA FOLLOWING THE SPIN-UP OF THE PULSAR*
}

\author{
D. B. MELROSE \\ Department of Theoretical Physics, Faculty of Science, \\ Australian National University, Canberra, Australia
}

\begin{abstract}
Observed enhanced activity in the central region of the Crab Nebula following the spin-up of the pulsar is discussed from the point of view of the transfer of energy to relativistic electrons. It is argued that a rapid deposition of energy associated with the spin-up of the pulsar causes a radial energy flux which becomes a flux in hydromagnetic activity at about the regions where enhanced synchrotron emission is observed. It is shown that such hydromagnetic activity is rapidly damped by the relativistic electrons with energy being transferred to the relativistic electrons. This acceleration can account for the short synchrotron halflifetimes observed. The model predicts highly enhanced $\mathrm{X}$-ray emission from the central region of the Nebula following a spin-up.
\end{abstract}

\section{Introduction}

Before the discovery of the pulsar in the Crab Nebula, two independent lines of reasoning led to the conclusion that an energy source was present.

Woltjer (1957) points out that the half-lifetime of the electrons radiating optical synchrotron is less than the age of the Nebula. The identification of the X-ray emission from the Nebula as synchrotron radiation indicates the presence of electrons with half-lifetimes as short as a year, e.g. less than the light travel time across the Nebula. This requires that these electrons either are being injected into the Nebula at the present time or are being accelerated in the Nebula at the present time. If the electrons are being accelerated rather than injected then simple arguments on the energy balance (Melrose, 1969) lead to the conclusion that the acceleration must be in a localized region. Furthermore, if one writes the rate of energy gain per electron in the form

$$
\left\langle\frac{\mathrm{d} E}{\mathrm{~d} t}\right\rangle=\frac{E}{T_{A}},
$$

where $T_{A}$ may be a function of the energy $E$ of the electron, then $T_{A}$ must be less than the synchrotron half-lifetime of the electron for this acceleration to have any significant effect. The power required in injected or accelerated electrons is about $10^{38} \mathrm{erg} \mathrm{sec}^{-1}$.

Scargle (1969) considers the so-called wisps in the central region of the Nebula and concludes that an energy source supplying a power comparable to that required to offset the synchrotron losses is required to account for the wisps. The wisps are interpreted as local enhancements in the synchrotron emission due to compressions of the magnetic field, see also Woltjer (1957), Shklovsky (1968). Although the three dimensional configuration of the compressions is unknown it appears that very strong com-

\footnotetext{
* This paper was presented by Dr. P. Stewart.
} 
pressions (magnetic field strength increasing severalfold) are required to give the observed enhancement in synchrotron emission. The wisps vary over time scales of the order of weeks to months in the central region of the Nebula. They propagate with a velocity of about $0.2 c$ which is interpreted as the velocity of propagation of the hydromagnetic compressional disturbance. Slower moving $\left(10^{-2} c\right)$ less intense enhancements in the synchrotron emission are observed in the outer regions of the Nebula. Scargle found that these enhancements vary quasi-periodically with a period of several years.

The discovery of the pulsar led to models for the electrodynamic acceleration of particles in the neighbourhood of the pulsar (Pacini, 1968; Ostriker and Gunn, 1968; Pacini and Rees, 1970). Such models may be able to account for the injection of relativistic electrons but do not obviously account for the excitation of the wisps.

Scargle and Harlan (1970) report increased activity in the wisps following the spin-up of the pulsar in September 1969. The increased activity at a given radial distance $r$ occurred approximately at a time $t=r / c$ where $t$ is the time elapsed from the spin-up. The energy associated with this enhanced activity was found to be between $10^{41}$ and $10^{45} \mathrm{erg}$, which is considerably greater than the change in the rotational energy of the pulsar resulting from the spin-up. Scargle and Harlan suggest that both the spin-up of the pulsar and the enhanced activity in the wisps result from an energetic event occurring in the neighbourhood of the pulsar. They suggest that the spinning of the pulsar builds up magnetic stresses in the surrounding magnetic field and that this magnetic energy is released by an instability. The quasi-periodic variations in the wisps suggest that such events occur quasi-periodically, consequently one would expect spin-ups of the pulsar to occur every several years.

The excitation of the wisps requires strong compressions of the magnetic field. Now, for example, an energy release of $10^{44} \mathrm{erg}$ into a volume of $10^{52} \mathrm{~cm}^{3}$ (about that inside the region where the wisps are observed) corresponds to an outward pressure which is greater than but comparable to the magnetic pressure in the field of $10^{-3}$ to $3 \times 10^{-4} \mathrm{G}$ in the region of the wisps. Thus the magnetic field in the region of the wisps would be strongly compressed by such an energy release.

These observations on the activity in the wisps indicate that the power requirements of the Nebula may be supplied through the quasi-periodic injection of energy into the region of the wisps in the form of strong localized compressions of the magnetic field. We assume this to be the case and consider the acceleration of electrons in the region of the wisps resulting from these compressions in the magnetic field. The basic requirements on the acceleration mechanism for it to be able to account for the observations are that the energy in the compressional disturbance be transferred to the relativistic electrons of a time scale of about a year and that the $T_{A}$ in (1) be less than the synchrotron halflifetimes of the electrons. We find that these requirements can be satisfied using parameters deduced from the observations of the wisps. This enhances the plausibility of the interpretation of the spin-up and the activity in the wisps as resulting from a quasi-periodic release of energy in the immediate surroundings of the pulsar. 


\section{Scattering and Acceleration of Particles}

In this section we give a semi-quantitative discussion of the acceleration of particles in the presence of periodic or quasi-periodic variations in the magnetic field strength. Acceleration results from the combination of two effects. If the magnetic field strength $B$ changes on a time scale much longer than the gyroperiods of the particles then the quantity $p_{\perp}^{2} / B$ is conserved ( $p_{\perp}=$ component of momentum perpendicular to $\mathbf{B}$ ), i.e. we have

$$
\frac{\mathrm{d} p_{\perp}^{2}}{\mathrm{~d} t}=\frac{p_{\perp}^{2}}{B} \frac{\mathrm{d} B}{\mathrm{~d} t} .
$$

Any initially isotropic distribution of particles becomes anisotropic. If the particles are scattered in any way, scattering time $\tau$ say, then this anisotropy is reduced. If the field varies between $B$ and $(1+\varepsilon) B$ over a characteristic time $T$ then the combination of these two effects leads to a mean rate of energy gain per particle given by

$$
\left\langle\frac{\mathrm{d} E}{\mathrm{~d} t}\right\rangle \sim \frac{\varepsilon^{2} \tau}{T^{2}} E \quad(\tau<T)
$$

to within a factor of order unity. Such acceleration is most effective for $\tau \approx T$ and goes to zero in both the limits $\tau \ll T$ and $\tau \gg T$.

The simplest example of such an acceleration is magnetic pumping. Suppose that $B$ varies according to

$$
\frac{\mathrm{d} B}{\mathrm{~d} t}=\frac{2 \varepsilon}{T} C B, \quad C= \begin{cases}+1 & \text { (compression) } \\ -1 & \text { (rarefaction) }\end{cases}
$$

i.e. that $B$ increases linearly with time from $B$ to $(1+\varepsilon) B$ in the first half-period $T / 2$ and then decreases linearly with time in the second half-period. In the first half-period the average value $\left\langle\sin ^{2} \alpha\right\rangle$ of $\left\langle p_{\perp}^{2}\right\rangle / p^{2}$ is greater than $\frac{2}{3}$ and in the second half period $\left\langle\sin ^{2} \alpha\right\rangle$ is less than $\frac{2}{3}$. We write

$$
\left\langle\sin ^{2} \alpha\right\rangle= \begin{cases}\frac{2}{3}+\delta & \text { (compression) } \\ \frac{2}{3}-\delta & \text { (rarefaction) }\end{cases}
$$

and note that when the tendency to become anisotropic is balanced by the tendency of the scattering to isotropize the particles (which balance is possible for $\tau<T$ ) we have

$$
\delta \sim \frac{2 \varepsilon \tau}{T}
$$

However according to (1) the rate of change of $p_{\perp}^{2}$ is proportional to $p^{2}\left\langle\sin ^{2} \alpha\right\rangle$ and so the increase in $p_{\perp}^{2}$ in the first half-period exceeds the decrease in the second halfperiod. The net energy gain over one period is given by (3) to within a factor of order unity. 
Magnetic pumping applies in particular to compressions propagating across the magnetic field. An analogous acceleration applies to compressions propagating along the magnetic field. Each such compression corresponds to a magnetic bottle which can trap some of the particles. Suppose that the compressions propagate with a velocity $V$ and have a length $\lambda=V T$. A fraction $\varepsilon$ of all particles approaching the neck of each bottle is reflected. There is an increase in the energy of the particle if the particle is reflected from an oncoming compression. There is a decrease in the energy of the particle if the particle overtakes the compression. However, as in Fermi acceleration, the particles are more likely to be reflected by meeting a compression head-on than on overtaking a compression. This leads to an average energy gain per particle of

$$
\Delta E \sim\left(\varepsilon \frac{V}{c}\right)^{2} E
$$

each time the particle reaches a compression (the factor $\varepsilon$ takes the probability of reflection into account). For $\tau \ll T$ relativistic particles diffuse the distance $\lambda$ between compressions in a time

$$
t \sim \frac{\lambda^{2}}{c^{2} \tau}, \quad \lambda=V T .
$$

The mean rate of energy gain, $\Delta E / t$, again reduces to (3) within a factor of order unity.

This type of acceleration can also be regarded as a form of viscous damping of the compressions. The anisotropy induced by the compressional disturbance is out of phase with the disturbance due to the scattering of the particles. If $\tau$ in (3) is independent of the energy $E$ then the rate of increase in the energy density in particles $W_{p}$ is

$$
\underset{\mathrm{d} t}{\mathrm{~d} W_{p}} \sim \frac{\varepsilon^{2} \tau}{T^{2}} W_{p}
$$

Conservation of energy implies that the energy density $W_{c}$ in the compressions decreases at the rate

$$
\frac{\mathrm{d} W_{c}}{\mathrm{~d} t}=-\frac{\mathrm{d} W_{p}}{\mathrm{~d} t} \sim-\frac{\varepsilon^{2} \tau}{T^{2}} W_{p},
$$

or equivalently

$$
\frac{1}{W_{c}} \frac{\mathrm{d} W_{c}}{\mathrm{~d} t} \sim-\frac{\tau}{T^{2}}\left(\frac{W_{p}}{W_{M}}\right),
$$

where $W_{c} \approx \varepsilon^{2} W_{M}$ with $W_{M}=$ ambient magnetic energy density.

The scattering of relativistic particles is discussed by a number of authors with reference to the scattering of cosmic rays, see Lerche (1967), Wentzel (1968), Kulsrud and Pearce (1969), Tademaru (1969) for example. The basic scattering process is associated with the fact that an anisotropic distribution of relativistic particles is 
unstable to the coherent emission of (relatively high frequency) hydromagnetic waves. The coherent emission of such waves reduces the anisotropy at a rate proportional to the energy density in these hydromagnetic waves. We analyze a particular example of this scattering in Section 3. Here we give a somewhat heuristic discussion of the effects of this scattering in the spirit of the discussion of the acceleration mechanisms given above.

The coherent emission of hydromagnetic waves requires that the anisotropy be greater than $v_{A} / c$, i.e. the hydromagnetic maser turns on only when the anisotropy, e.g.

$$
\delta=\frac{|f(p, \alpha=\pi / 2)-f(p, \alpha=0)|}{f(p, \alpha=\pi / 2)+f(p, \alpha=0)}
$$

exceeds $v_{A} / c$ where $v_{A}=$ Alfvén velocity. This requires $\varepsilon>v_{A} / c$ for any compression to cause coherent emission of waves. Let us suppose the growth time for the waves is very much shorter than the period $T$ of the compressions. Then the growth of the waves will proceed until the anisotropy is maintained near its minimum possible value, i.e. we then have an anisotropy

$$
\delta \approx \frac{v_{A}}{c} \approx \frac{2 \varepsilon \tau}{T}
$$

The implied effective scattering time is

$$
\tau \sim \frac{v_{A}}{c} \frac{T}{\varepsilon}
$$

The acceleration (3) with this scattering leads to an acceleration described by

$$
\left\langle\frac{\mathrm{d} E}{\mathrm{~d} t}\right\rangle \sim \frac{v_{A}}{\frac{\varepsilon}{c}} \frac{\varepsilon}{T} E
$$

to within a factor of order unity. In Section 3 we derive the appropriate numerical factor for one particular situation.

In the region of the wisps the parameters $v_{A}, \varepsilon, T, W_{p}, W_{M}$ can all be estimated from the observations. It is already clear from (7) that for $T \sim$ one month, $v_{A} \sim 0.2 c$, $\varepsilon \sim 1$ the acceleration time $T_{A}$ in (1) is of the order of a year as required. Furthermore we then infer from (5) and (6), with $W_{p} \sim W_{M}$ from observation, that the wisps would damp out over about a year, as observed, transferring their energy to the relativistic electrons. Our more detailed discussion in Section 3 is aimed at estimating the numerical factor in (7) and at deducing the conditions under which the growth of the waves is rapid enough to maintain the anisotropy at a value of order $v_{A} / c$.

\section{Magnetic Pumping}

We analyze the acceleration of relativistic particles due to magnetic pumping in the case where the field strength varies linearly with time according to Equation (4). We 
refer the interested reader to Melrose and Wentzel (1970) for a more detailed discussion of the details of the equations describing the growth of the waves and the scattering by the waves and to Melrose (1970) for the details of the expansion in spherical harmonics used below.

We assume that the relativistic electrons have a number density $n$ and a distribution function

$$
f(p)=\frac{(a-3)}{4 \pi} p_{0}^{-3}\left(\frac{p}{p_{0}}\right)^{-a} \quad\left(a>3, p>p_{0}\right) .
$$

Note that $f(p) \propto p^{-a}$ corresponds to an energy distribution $N(E) \propto E^{2-a}$.

With $\mu=\cos$ (pitch angle), the change in the distribution function due to the changes described by (4) is given by

$$
\begin{aligned}
\left.\frac{\partial}{\partial t} f(p, \mu)\right|_{C} & =-\frac{\partial}{\partial p_{\perp}^{2}}\left[2 \frac{\varepsilon C}{T} p_{\perp}^{2} f(p, \mu)\right] \\
& =-\frac{C \varepsilon}{T}\left(\frac{1}{p} \frac{\partial}{\partial p}-\frac{\mu}{p^{2}} \frac{\partial}{\partial \mu}\right)\left\{p^{2}\left(1-\mu^{2}\right) f(p, \mu)\right\}
\end{aligned}
$$

The anisotropy induced in this way is such that $f(p, \mu)$ is an even function of $\mu$. Thus if we expand in spherical harmonics by writing

$$
f(p, \mu)=\sum_{n=0}^{\infty} f_{n}(p) P_{n}(\mu)
$$

where $P_{n}$ is a Legendre polynomial, then $f_{n}$ is non-zero only for even $n$.

Anisotropies which are even in $\mu$ lead to coherent emission of circularly polarized hydromagnetic waves rather than linearly polarized hydromagnetic waves, see Melrose and Wentzel (1970). To an excellent approximation these waves may be regarded as propagating exactly along the magnetic field lines. According to Melrose and Wentzel (1970) a distribution of electrons of the form (8) subjected to a compression $(C=+1)$ reduced by either emitting or absorbing waves of one handedness or the other. If $\Gamma=+1(-1)$ denotes absorption (emission) and $P=+1(-1)$ denotes right (left) hand circularly polarized waves then the condition for the anisotropy to be reduced is

$$
C P \Gamma=-1 .
$$

If $k$ denotes the component of the wave number along the field lines, then electrons with a given sign of $\mu$ interact with waves with a given sign of $k$ only if the condition

$$
\frac{\mu}{|\mu||k|} \frac{k}{|k|}=-P
$$

is satisfied.

Consider the following sequence of processes. Suppose that at the start of a compression $(C=+1)$ waves of both handedness $P=+1$ are already present. According to (11) the anisotropy induced by the compression can be reduced by absorbing $(\Gamma=+1)$ the waves with $P=-1$. The anisotropy can also be reduced by the emission 
$(\Gamma=-1)$ of waves with $P=-1$. Both processes occur. As the compression proceeds the ratio of the energy density in the waves with $P=-1$ to that in waves with $P=+1$ decreases. At the end of the compression phase most of the waves have polarization $P=+1$. When rarefaction $(C=-1)$ begins the anisotropy is reduced primarily by absorbing $(\Gamma=+1)$ these waves $(P=+1)$. As the rarefaction phase proceeds the ratio of the energy density in waves with $P=+1$ to that in waves with $P=-1$ decreases. At the end of the rarefaction phase most of the waves have handedness $P=-1$. The cycle then recommences.

This sequence of processes allows the anisotropy to remain small at every phase of the cycle. Any intrinsic damping of the waves has little effect provided that this damping is not great enough to prevent the growth of the waves. The only essential condition for this sequence of events to occur during cyclic variations of the field strength is that the waves grow and damp on a time scale much shorter than the period of compression.

Let $W(k)$ be the energy density in the waves in the range $\mathrm{d} k$ at $k$. We do not distinguish explicitly between waves of different handedness because the handedness is more easily discussed independently as above. The following equations describe the growth or damping of the waves and the scattering of the particles by the waves:

$$
\begin{aligned}
& \frac{\partial}{\partial t} W(k)=-\gamma(k) W(k), \\
& \gamma(k)=\frac{-2 \pi^{2} e^{2} v_{A} n}{|k| c} \int_{-1}^{+1} \mathrm{~d} \mu \frac{\left(1-\mu^{2}\right)}{|\mu|} \\
& \times\left[p^{2}\left\{\frac{k}{|k| \partial \mu} f(p, \mu)+\frac{v_{A}}{c} p \frac{\partial}{\partial p} f(p, \mu)\right]_{p=p_{R}},\right. \\
& p_{R}=\frac{p_{0} k_{0}}{|k \mu|}, \quad p_{0} k_{0}=\frac{|e| B}{c} \text {; } \\
& \left.\frac{\partial}{\partial t} f(p, \mu)\right|_{s}=\frac{\partial}{\partial \mu}\left[D(p, \mu)\left\{\frac{\partial}{\partial \mu} f(p, \mu)+\frac{k}{|k|} \frac{v_{A}}{c} p \frac{\partial}{\partial p} f(p, \mu)\right\}\right], \\
& D(p, \mu)=\frac{\pi e^{2}\left(1-\mu^{2}\right)}{p^{2} c|\mu|} W\left(k_{R}\right), \\
& k_{R}=\frac{p_{0} k_{0}}{p|\mu|} \text {. }
\end{aligned}
$$

The sign $k /|k|$ is determined by (11) and (12) with $\Gamma=\gamma(k) /|\gamma(k)|$.

It is impractical to attempt to follow the time development explicitly. However one can readily show by expanding (9) in spherical harmonics that the term $f_{2}(p)$ in (10) is the first to grow. Initially only this term need be considered in (14) and (16). As indicated by our discussion above, waves are always present once the cycle is initiated. 
Keeping only the term $f_{2}(p)$ in the expansions of (9), (14) and (16) leads to a selfconsistency problem in which the values of $f_{2}(p)$ and $W(k)$ are to be determined by the condition that the reduction of $f_{2}(p)$, due to scattering, balances the growth of $f_{2}(p)$, due to the change in the field strength. An important part of the solution of this self-consistency problem is the determination of the $k$-dependence of $W(k)$.

Expanding (14) and (16) in spherical harmonics and retaining only the lowest order non-trivial terms gives

$$
\begin{aligned}
\left.\frac{\partial}{\partial t} f_{0}(p)\right|_{c} & =-C \frac{\varepsilon}{T}(a-3)\left\{\frac{2}{3} f_{0}(p)-\frac{2}{15} f_{2}(p)\right\} \\
\left.\frac{\partial}{\partial t} f_{2}(p)\right|_{c} & =-C \frac{\varepsilon}{T} \frac{2 a}{3} f_{0}(p) \\
\left.\frac{\partial}{\partial t} f_{2}(p)\right|_{s} & =-\tau_{1}^{-1} f_{2}(p)-a C \Gamma \frac{v_{A}}{c} \tau_{2}^{-1} f_{0}(p)
\end{aligned}
$$

where we use (8) and where

$$
\begin{aligned}
& \tau_{1}^{-1}(p)=\frac{5}{2} \int_{-1}^{+1} \mathrm{~d} \mu(3 \mu)^{2} D(p, \mu), \\
& \tau_{2}^{-1}(p)=\frac{5}{2} \int_{-1}^{+1} \mathrm{~d} \mu \frac{\mu}{|\mu|}(3 \mu) D(p, \mu) .
\end{aligned}
$$

By requiring

$$
\left.\frac{\partial}{\partial t} f_{2}(p)\right|_{c}+\left.\frac{\partial}{\partial t} f_{2}(p)\right|_{s}=0,
$$

(20) and (21) allow one to find $f_{2}(p)$. On inserting this value of $f_{2}(p)$ in (13) with (14) one finds

$$
\begin{aligned}
& \frac{\partial}{\partial t} W(k)=-\frac{1}{T_{0}} W(k)+C P \frac{A}{T_{1}} \\
& T_{0}^{-1} \propto\left(\frac{k}{k_{0}}\right)^{a-3}, \quad A T_{1}^{-1} \propto\left(\frac{k}{k_{0}}\right)^{a-5} .
\end{aligned}
$$

For $T_{0} \ll T$ the magnitude of $W(k)$ does not vary rapidly over a period of the compression; the variation of $W(k)$ with time is sketched in Figure 1 . The mean value of $W(k)$ for $T_{0} \ll T$ is

$$
\langle W(k)\rangle \approx \frac{A T_{0}}{T_{1}} \propto\left(\frac{k}{k_{0}}\right)^{-2} .
$$

This identification of the functional form of $W(k)$ allows us to determine the solution of the self-consistency problem. One can then proceed to consider the inclusion of 


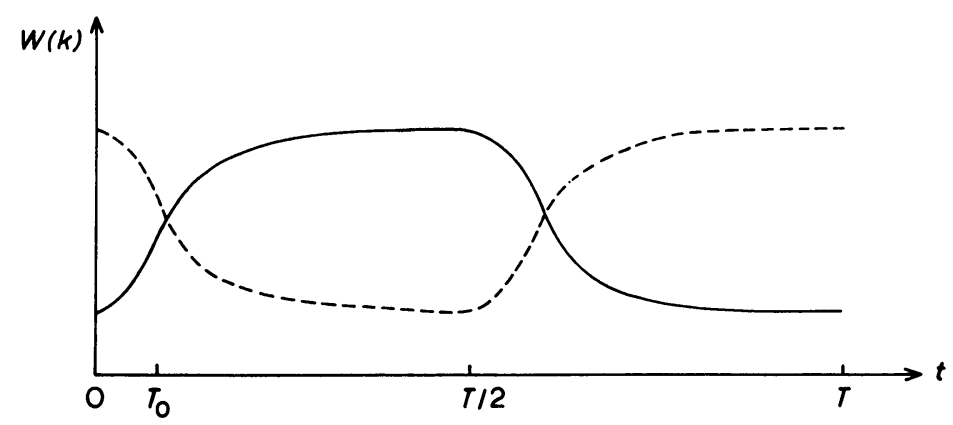

Fig. 1. $W(k)$ for waves of both handedness is sketched as a function of time over a period $T$ of a compressional disturbance with time $t=0$ corresponding to the onset of compression. The time $T_{0} \ll T$ is indicated.

the term $f_{4}(p)$, resolve the problem and so on. However, as in the analogous problem discussed in Melrose (1970), the inclusion of more terms in the expansion in spherical harmonics leads to only minor numerical changes in the final result.

Retaining only the term $f_{2}(p)$ gives

$$
\begin{aligned}
W(k)= & W_{0}\left(\frac{k}{k_{0}}\right)^{-2} \quad\left(k<k_{0}\right), \\
W_{0} & =\frac{8}{15} \frac{p_{0}^{2} C}{\pi^{2} e^{2}} \frac{a(a-2) c}{a^{2}-1} \frac{\varepsilon}{v_{A}} \frac{1}{T}, \\
\tau_{1}^{-1} & =\frac{15}{4} D_{0}, \quad \tau_{2}^{-1}=2 D_{0}, \\
D_{0}= & \frac{\pi^{2} e^{2}}{p_{0}^{2} c} W_{0}=\frac{8}{15} \frac{a(a-2)}{a^{2}-1} \frac{\varepsilon}{v_{A}} \frac{\varepsilon}{T}, \\
& \frac{f_{2}(p)}{f_{0}(p)}=-C \frac{a^{2}-1}{3(a-2)} \frac{v_{A}}{c}, \\
\frac{1}{T_{0}}= & \frac{2 \pi^{2} e^{2} n}{p_{0} k_{0}} \frac{(a-3)}{(a-2)}\left(\frac{v_{A}}{c}\right)^{2}\left(\frac{k}{k_{0}}\right)^{a-3} .
\end{aligned}
$$

Inserting the value of $f_{2}(p)$ so determined in (19) one finds that the rate of change of $f_{0}(p)$ includes the term

$$
\left\langle\frac{\partial}{\partial t} f_{0}(p)\right\rangle=\frac{2}{45} \frac{(a-3)\left(a^{2}-1\right)}{a-2} \frac{v_{A}}{c} \frac{\varepsilon}{T} f_{0}(p)
$$

which has the same sign for both compression and rarefaction. This term describes magnetic pumping. In fact (25) leads to an acceleration of the form (1) with

$$
T_{A}=\frac{45}{2} \frac{(a-2)}{\left(a^{2}-1\right)(a-3)}\left(\frac{c}{v_{A}}\right)\left(\frac{T}{\varepsilon}\right) \text {. }
$$


The scattering times $\tau_{1}$ and $\tau_{2}$ in (24) are of the form (6) which we deduced from qualitative arguments. Similarly the acceleration (1) with (26) is of the form (7) but (26) includes an estimation of the undetermined factor of order unity in (7).

The condition for the above analysis to be applicable is that the time $T_{0}$, which is characteristic of the growth or damping of the waves due to the relativistic electrons, be much less than the time $T$. If the waves are intrinsically damped in the background plasma at a rate

$$
\frac{\partial}{\partial t} W(k)=-\frac{1}{T_{D}} W(k)
$$

then the above analysis applies only if

$$
T_{0}<\min \left[T, T_{D}\right]
$$

The condition $T_{0}<T$ with $a<3$ leads to an upper limit on the momentum of relativistic particles which can be accelerated by this process. This follows by noting the fact that particles with a given $p$ interact with waves over a small range of $k$, and vice versa, around

$$
p k \approx p_{0} k_{0} .
$$

The inequality (28) need not be extreme because for $T_{0} \approx T$ the scattering time $\tau$ is also of the order of $T$. The acceleration (3) becomes more effective as the inequality $T_{0} \ll T$ starts to break down. Only for $T_{0}>T$ does the acceleration become ineffective.

\section{Discussion}

If the transfer of energy from the pulsar to the relativistic electrons occurs by the intermediate excitation of the wisps then a number of conditions need to be satisfied. Firstly, the time averaged power input into the wisps must balance the time averaged power lost by the Nebula (primarily synchrotron losses). Secondly, the acceleration must cause the energy per electron to increase faster than this energy decreases due to synchrotron losses, e.g. $T_{A}$ in (1) must be of the order of a year. Thirdly, the electrons must remain in the region where acceleration occurs for a sufficiently long time, e.g. a year, to be significantly accelerated. Fourthly, the electrons must be accelerated to a sufficiently high energy $\left(>10^{14} \mathrm{eV}\right)$ to account for the highest observed frequencies in the synchrotron emission. Fifthly, the wisps must lose their energy to relativistic electrons over a time of a year or so.

The energetic considerations remain uncertain because any estimation of the power input into the wisps depends on geometric assumptions. Only a surface brightness is observed; one must make an assumption on the configuration in three dimensions. All that can be said is that the mean power input into the wisps is consistent with this being the source of the mean power lost through synchrotron radiation.

The acceleration time $T_{A}$, see (26), with $a=4, \varepsilon \approx 1$ to $3, T \approx 3 \times 10^{6} \mathrm{sec}, v_{A} \approx 0.2 c$ from observations of the wisps, is of the order of a year. With these numbers the 
scattering time (6) is of the order of $3 \times 10^{5} \mathrm{sec}$ and so electrons diffuse over a distance of $10^{17} \mathrm{~cm}$ (to escape from the region where acceleration occurs) in a time

$$
t \approx \frac{\left(10^{17}\right)^{2}}{c^{2}\left(3 \times 10^{5}\right)} \approx 3 \times 10^{7} \mathrm{sec} .
$$

Thus individual electrons remain in the region where acceleration occurs for about a year.

The maximum energy $\left(E_{\max }=p_{\max } c\right)$ to which electrons can be accelerated follows from (28) and (29) with $T_{0}$ given by (24). If we set $p_{0} \approx 10^{11} \mathrm{eV} / c$ in (8) thereby restricting our attention to the electrons radiating at optical and higher frequencies (these require continued acceleration) then the observed power output requires $n \approx 10^{-7} \mathrm{~cm}^{-3}$ for these electrons. With $a=4$ and $T=3 \times 10^{6} \mathrm{sec}$ the condition $T_{0}<T$ requires

$$
\frac{p}{p_{0}}<\frac{2 \pi^{2} e^{2} n c T}{|e| B} \frac{(a-3)}{(a-2)}\left(\frac{v_{A}}{c}\right)^{2} \approx 6 \times 10^{3},
$$

where we set $B=3 \times 10^{-4} \mathrm{G}$. Thus $T_{0}<T$ requires $E<E_{\max } \approx 6 \times 10^{14} \mathrm{eV}$, i.e. electrons can be accelerated to energies in excess of $10^{14} \mathrm{eV}$ as required.

It is believed that there is approximate equipartition of energy $\left(W \approx W_{M}\right)$ in the Nebula. With equipartition equation (5) predicts that the wisps damp out over a time $T^{2} / \tau \approx 3 \times 10^{7} \sec \approx 1 \mathrm{yr}$. Thus it seems plausible that all the conditions listed above are satisfied by the processes under discussion.

The interpretation of the wisps as compressions and the above discussion of the acceleration processes associated with such compressions requires that $\mathrm{X}$-ray emission be strongly enhanced following enhanced activity in the wisps. This results from two effects. Firstly the enhancement in surface brightness due to a compression is greater at X-ray frequencies than at optical frequencies. Secondly the shortness of the halflifetimes of electrons radiating in the hard X-ray ( $\gtrsim 100 \mathrm{keV})$ region of the spectrum implies that this emission should be observed to die away in between periods of enhanced activity in the wisps. The acceleration envisaged here implies an upsurge in hard X-ray emission for about a year during enhanced activity in the wisps, then followed by a decrease in hard $X$-ray emission until the next period of enhanced activity. Furthermore the hard X-ray emission can arise only from the central region of the Nebula.

\section{References}

Kulsrud, R. M. and Pearce, W. D.: 1969, Astrophys. J. 156, 445.

Lerche, I.: 1967, Astrophys. J. 147, 689.

Melrose, D. B.: 1969, Astrophys. Space Sci. 4, 165.

Melrose, D. B.: 1970, Astrophys. Space Sci. 6, 321.

Melrose, D. B. and Wentzel, D. G.: 1970, Astrophys. J. 161, 457.

Ostriker, J. and Gunn, J.: 1969, Astrophys. J. 157, 1395.

Pacini, F.: 1968, Nature 219, 145.

Pacini, F. and Rees, M. J.: 1970, Nature 226, 622.

Scargle, J. D.: 1969, Astrophys. J. 156, 401. 
Scargle, J. D. and Harlan, E. A.: 1970, Contributions from Lick Observatory No. 313.

Shklovsky, I. S.: 1969, Supernovae, Wiley-Interscience Publ., New York.

Tademaru, E.: 1969, Astrophys. J. 158, 959.

Wentzel, D. G.: 1968, Astrophys. J. 156, 303.

Woltjer, L.: 1957, Bull. Astron. Inst. Neth. 14, 39.

\section{Discussion}

I. Lerche: The result obtained must depend critically on the initial conditions which were assumed. P. Stewart: Dr. Melrose assumed a wave distribution proportional to $\left(k / k_{0}\right)^{-2}$.

I. Lerche: There is no definite evidence for the distribution and it must be regarded as an assumption.

J. P. Ostriker: Electrons will tend to lose energy while protons will not. Hence there must be a build up of proton numbers.

P. Stewart: This makes the time scale for energy loss rather imporiant.

F. C. Michel: How much compression does Melrose expect?

P. Stewart: A factor of 2 or 3.

F. C. Michel: This leads to acceleration by the same factor.

J. E. Felten: Would you expect the X-ray output to vary over a period of a year?

P. Stewart: Yes. Melrose makes the point that the X-rays will vary more than the light, where the particle lifetimes are longer. It even seems possible that the radio emission from the Crab Nebula changes in one month; if so, then the supersynthesis map will be incorrect.

A. S. Wilson: The beam size in the Cambridge maps corresponds to two months motion at the velocity of light. Any changes in the emission are unlikely to have any effect on the maps.

$R$. G. Conway: The observations at Green Bank took six months but there is no evidence for any changes during that time. 